\title{
Probability estimation of ignitions formation in the electrical network
}

\author{
Sergey Solyonyj*, Vladislav Shishlakov and Oksana Solenaya \\ SUAI, Department of Electromechanics and Robotics, 190000 Saint-Petersburg, Bolshaya Morskaya str., 67, Russia
}

\begin{abstract}
Their fire safety is one of the major requirements, which is imposed to electric networks. However extensiveness of methods of her assessment and a different of approaches to definition of factors of danger leads to complication of the choice of a technique and process of an assessment. The work purpose is development of a uniform method of mathematical modelling and an assessment of safe operation of electric networks.
\end{abstract}

\section{Introduction}

Within the Federal target program «Fire Safety in the Russian Federation for the Period till 2017» № 1481 from 12.30.2012, carrying out researches in the field of development of mathematical models and methods by definition and forecasting of probability of emergence of the fires, effective ways of their prevention and mitigation of consequences is provided. It also agrees, to the Federal law of the Russian Federation № 117-FL «About introduction of amendments to the Federal law № 123-FL «Technical regulations about requirements of fire safety» from 7.10.2012 [1], development of the new technical solutions and organizational actions, use operating and creation of new normative documents which application will allow to ensure fire safety of the industrial and household electrified facilities is provided.

The analysis of statistics of the fires in the Russian Federation because of damages of electric conducting from 2006 for 2016 [2] demonstrates that if not to develop new and not to improve the known methods, ways of ensuring safe operation of systems of lowvoltage power supply, then in them to confidential probability 0,95 there will be about 42000 fires from the electrotechnical reasons [3].

Therefore, the work directed to creation new and improvement of the known mathematical models, methods of an assessment and forecasting of safe operation of systems of low-voltage power supply, and also development of new technical and organizational actions for improvement of a complex of means of protection, low-voltage electric network up to $1 \mathrm{kV}$, is an urgent scientific and technical task which solution will allow not to allow cases of emergence of ignitions of combustible material (isolation, etc.) in systems of lowvoltage power supply and will ensure their fire safety at the level of the existing normative documents [4].

Due to the adoption of laws $[1,5]$ special relevance is acquired development and deployment in domestic practice of evidence-based recommendations about ensuring fire safety of systems of low-voltage power supply, use of methods by means of which the opportunity of an assessment of the existing level of fire danger is given and to compare him to the value $110^{-6}$, which is legislatively established state standard [4].

\section{Problem definition}

We will assume that ignition of combustible material on an electrical network because of damage of electrical wiring happens in case of coincidence in space and time of the following three processes: there was a damage of an electrical network which is energized; the system of protection refused actuating; there was a combustible material near the place of damage of an electrical network [6-8].

We will provide state change of an electrical network in the form of uniform Markov process of $\alpha(t)$ with two discrete states and the continuous time eventually. We will designate through « 0 » it a status of an electrical network in which damages of its insulation aren't watched, and through «1»- duration of time in which there is an electrical network after damage of its insulation (protection reaction time).

We will designate parameters of process $\alpha(t)$ through $\lambda_{1}$ and $\mu_{1}$ where $\lambda_{1}$ characterizes intensity or speed of state change of an electrical network, frequency of transitions from a status « $0 »$ in a status $« 1 »$, and $\mu_{l}-$ is characterized by the frequency of transitions from a status «1» in a status " 0 ".

We will designate through $\beta(t)$ uniform Markov accidental process with two discrete states $-\langle 0 »$ and «1» and the continuous time which characterizes state change of system of protection: «0» - up state of system of protection (the waiting mode) and «1»- the damaged status of system (comes to light only as a result of diagnostics). Parameters of process $\beta(t)$ following: $\lambda_{I}-$ the speed of change of operable periods (working state

\footnotetext{
*Corresponding author: ssv555ssv@yandex.ru
} 
of system of protection) on no serviceable; $\mu_{2}-$ the speed of change of no serviceable periods during which the system of protection was in the refused status, on operable. Diagnostics of system of protection is carried out through each $\Theta_{l}$ of year.

Existence or absence of combustible material in close proximity to accidental insulation failure of an electrical network it is representable similar uniform markov process of $\xi(t)$ with two discrete states: «0»- near an electrical network which is energized there is no combustible material (dust, paper, liquid flammable materials, etc.) and «1»- near a spacer of an electrical network there is a combustible material.

We will designate parameters of process $\xi(t)$ through $\lambda_{3}$ and $\mu_{3}$ where $\lambda_{3}-$ the frequency of appearance of combustible material in close proximity to an electrical network, and characterizes by $\mu_{3}$ the speed of change of availability of combustible material with its complete or partial elimination which will provide a fire safety of a securable object.

Availability of combustible material near a spacer of conductors of an electrical network or its customers comes to light and eliminated as a result of conducting preventive checks with $\Theta_{2}$ time interval.

Ignition of combustible material on an electrical network will happen at the time of coincidence in space and time of three accidental processes of $\alpha(t), \beta(t)$ and $\xi(t)$ is able 1 that is when: $\alpha(t)=1, \beta(t)=1, \xi(t)=1$.

We will assume that in initial time point of $\alpha(t)=0$, $\beta(t)=0, \xi(t)=0$ and parameters of processes $\alpha(t), \beta(t), \xi(t)$, that is their characteristics $\lambda_{1} ; \mu_{1}, \lambda_{2} ; \mu_{2}, \lambda_{3} ; \mu_{3}$ are known.

The task consists in that knowing parameters of processes $\lambda_{1} ; \mu_{1}, \lambda_{2} ; \mu_{2}, \lambda_{3} ; \mu_{3}$ to define: average time before the first ignition of $\tau_{l}$, dispersion of this time of $D_{l}$, probability of finding of electric network in each of possible conditions of $P_{i}(t)$, probability of ignition of combustible material in electric network $F(t)$.

\section{Assessment of safe operation}

We will consider set of processes of $\alpha(t), \beta(t)$ and $\xi(t)$ as one regular uniform Markov process of $\alpha(t)$ with eight discrete states and continuous time (Fig. 1: $\zeta_{k}$-duration of finding of process of $\alpha e(t)$ in each of eight possible states $k=1 \div 8 ; \alpha(t), \beta(t)$, and $\xi(t)$ - uniform casual Markov processes with two discrete states and continuous time; $\alpha_{i}^{(0)}, \alpha_{j}^{(l)}, \beta_{i}^{(0)}, \beta_{j}^{(l)}, \xi_{i}^{(0)}, \xi_{j}^{(I)}-$ time intervals between events and their duration $i, j=1 \div n ; \Theta_{l}$, $\Theta_{2}$ - time intervals between diagnostics of system of protection and verifications of combustible material near emergence of a dangerous electric source in electric network; $\tau_{0}{ }^{1}$ - time before the first ignition of combustible material in electric network).

At any moment process of $\alpha(t)$ can be in one of eight possible discrete states: $e_{1}(0,0,0), e_{2}(1,0,0), e_{3}(0,1,0)$, $e_{4}(0,0,1), e_{5}(1,0,1), e_{6}(0,1,1), e_{7}(1,1,0), e_{8}(1,1,1)$.

The condition of $e_{8}(1,1,1)$ absorbing. Having got to this state, the system can't leave it. The being able $e_{8}(1,1,1)$ occurs ignition of combustible material.

We will designate through $\zeta_{k}, k=1 \div 8$, time of stay of process of $\alpha(t)$ is able $e_{k}$. For regular uniform Markov process of $\alpha(t)$ with discrete number of states and continuous time random variables of $\zeta_{k}$ don't contradict an exponential function of distribution of probabilities:

$$
F_{\zeta}(t)=P\{\zeta<t\}=1-e^{-\lambda}{ }_{i i} t
$$

We will designate through $P_{i i}(\Delta t)$ probability that process of $\alpha(t)$ for a small period of $\Delta t$ will remain to the being able $e_{i}, i=1 \div 8$, and through $P_{i j}(\Delta t)$ - probability that the system in time $\Delta t$ will pass from $e_{i}$ a state into a condition of $e_{j}, j=1 \div 8$.

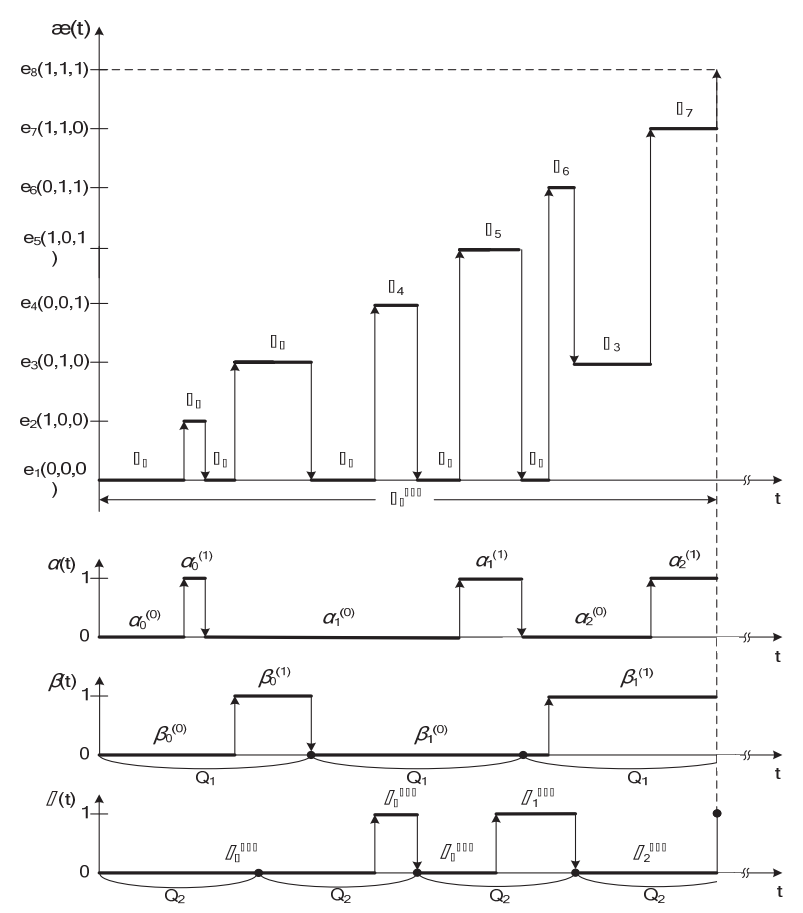

Fig. 1. Possible realization of uniform casual Markov process with eight discrete states and continuous time.

The probability of transitions of $P_{i i}(\Delta t)$ and $P_{i j}(\Delta t)$ decides on the help of formulas:

$P_{i i}(\Delta t)=P\left[c e(t+\Delta t)=e_{i} / \propto e(t)=e_{i}\right]=P\left\{e_{i} \rightarrow e_{i}\right\}=1-\lambda_{i i} \Delta t+0(\Delta t)$,

$P_{i j}(\Delta t)=P\left[a(t+\Delta t)=e_{j} / \propto e(t)=e_{j}\right]=P\left\{e_{j} \rightarrow e_{j}\right\}=1-\lambda_{i j} \Delta t+0(\Delta t)$

In a formula (2) the size $\lambda_{i i} \Delta t$ considers to within the second order of a trifle that during $\Delta t$ there will be no transition of system from a condition of $e_{i}$ in a condition of $e_{j}$, that is process will remain to the being able $e_{i}$.

Follows from a formula (3) that the size $\lambda_{i j} \Delta t$ considers to within members of the second order of a trifle that during $\Delta t$ there will be a transition of system from a condition of $e_{i}$ in a condition of $e_{j}$.

Using a matrix of probabilities of transitions $P(\Delta t)[6]$ we find a matrix $A$ :

$$
A=\lim _{\Delta t \rightarrow 0} \frac{Q(\Delta t)-I}{\Delta t}
$$

where $I-$ a single matrix.

The matrix of $Q(\Delta t)$ is from $P(\Delta t)$ matrix by an exception of the absorbing state - the last line and the last column. 
Relation limit $O(\Delta t)$ to $\Delta t$ at $\Delta t$ aspiring to «0» is equal «0»:

$$
\lim _{\Delta t \rightarrow 0} \frac{0(\Delta t)}{\Delta t}=0
$$

Using matrix of probabilities of transitions $P(\Delta t)$, (4) and (5), we will receive a matrix $\mathrm{A}\left(c_{1}=\lambda_{1}+\lambda_{2}+\lambda_{3}\right.$, $c_{2}=\mu_{1}+\lambda_{2}+\lambda_{3}, c_{3}=\lambda_{1}+\mu_{3}+\lambda_{3}, c_{4}=\lambda_{1}+\lambda_{2}+\mu_{3}, c_{5}=\mu_{1}+\lambda_{2}+\mu_{3}$, $\left.c_{6}=\lambda_{1}+\mu_{2}+\mu_{3}, c_{7}=\mu_{1}+\mu_{2}+\lambda_{3}\right)$.

$$
A=\left(\begin{array}{ccccccc}
-c_{1} & \lambda_{1} & \lambda_{2} & \lambda_{3} & 0 & 0 & 0 \\
\mu_{1} & -c_{2} & 0 & 0 & \lambda_{3} & 0 & \lambda_{2} \\
\mu_{2} & 0 & -c_{3} & 0 & 0 & \lambda_{3} & \lambda_{1} \\
\mu_{3} & 0 & 0 & -c_{4} & \lambda_{1} & \lambda_{2} & 0 \\
0 & \mu_{3} & 0 & \mu_{1} & -c_{5} & 0 & 0 \\
0 & 0 & \mu_{3} & \mu_{2} & 0 & -c_{6} & 0 \\
0 & \mu_{2} & \mu_{1} & 0 & 0 & 0 & -c_{7}
\end{array}\right) .
$$

We will find a matrix of $N$ as follows:

$$
N=\lim _{\Delta t \rightarrow 0} \frac{I-Q(\Delta t)}{\Delta t}
$$

Using matrix of probabilities of transitions $P(\Delta t),(5)$ and (7), we find:

$$
N=\left(\begin{array}{ccccccc}
c_{1} & -\lambda_{1} & -\lambda_{2} & -\lambda_{3} & 0 & 0 & 0 \\
-\mu_{1} & c_{2} & 0 & 0 & -\lambda_{3} & 0 & -\lambda_{2} \\
-\mu_{2} & 0 & c_{3} & 0 & 0 & -\lambda_{3} & -\lambda_{1} \\
-\mu_{3} & 0 & 0 & c_{4} & -\lambda_{1} & -\lambda_{2} & 0 \\
0 & -\mu_{3} & 0 & -\mu_{1} & c_{5} & 0 & 0 \\
0 & 0 & -\mu_{3} & -\mu_{2} & 0 & c_{6} & 0 \\
0 & -\mu_{2} & -\mu_{1} & 0 & 0 & 0 & c_{7}
\end{array}\right),
$$

The average time of $\tau_{1}$ before emergence of ignition of combustible material in electric network, dispersion of $D_{l}$ and function of probability $P^{\prime}(t)$ of finding of system during $t$ time in each of $i$ of possible states, $i=1 \div r$, it is possible to find using systems of the equations:

$$
\begin{gathered}
\tau=N^{-1} \xi, \\
D=(2 N-I) \tau-\tau^{2}, \\
P^{\prime}(t)=P(t) A,
\end{gathered}
$$

where $\xi$ - a vector column which all elements are equal to $1 ; \tau$ - vector column $\tau=\left[\tau_{i}\right]_{i=1}^{7} ; \tau^{2}-\tau^{2}=\left[\tau^{2}{ }_{i}\right]^{7}{ }_{i=1}$ vector column; $P^{\prime}(t)$ - vector line $P^{\prime}(t)=\left[P_{i}^{\prime}(t)\right]^{7}{ }_{i=1} ; P(t)-$ vector line $P(t)=\left[P_{i}(t)\right]_{i=1}^{7}$.

Using a matrix (8) and system of the algebraic equations (9), we will write down system of the equations for finding of $\tau_{l}$ in a look:

$$
\left(\begin{array}{c}
\tau_{1} \\
\tau_{2} \\
\tau_{3} \\
\tau_{4} \\
\tau_{5} \\
\tau_{6} \\
\tau_{7}
\end{array}\right)=\left(\begin{array}{ccccccc}
c_{1} & -\lambda_{1} & -\lambda_{2} & -\lambda_{3} & 0 & 0 & 0 \\
-\mu_{1} & c_{2} & 0 & 0 & -\lambda_{3} & 0 & -\lambda_{2} \\
-\mu_{2} & 0 & c_{3} & 0 & 0 & -\lambda_{3} & -\lambda_{1} \\
-\mu_{3} & 0 & 0 & c_{4} & -\lambda_{1} & -\lambda_{2} & 0 \\
0 & -\mu_{3} & 0 & -\mu_{1} & c_{5} & 0 & 0 \\
0 & 0 & -\mu_{3} & -\mu_{2} & 0 & c_{6} & 0 \\
0 & -\mu_{2} & -\mu_{1} & 0 & 0 & 0 & c_{7}
\end{array}\right)^{-1} \cdot\left(\begin{array}{l}
1 \\
1 \\
1 \\
1 \\
1 \\
1 \\
1
\end{array}\right) \cdot(12)
$$

Using a matrix (8) and system of the equations (10) we find dispersion of $D_{l}$ from system of the equations of a look:

$$
\left(\begin{array}{l}
D_{1} \\
D_{2} \\
D_{3} \\
D_{4} \\
D_{5} \\
D_{6} \\
D_{7}
\end{array}\right) \cdot\left(\begin{array}{ccccccc}
c_{1} & -\lambda_{1} & -\lambda_{2} & -\lambda_{3} & 0 & 0 & 0 \\
-\mu_{1} & c_{2} & 0 & 0 & -\lambda_{3} & 0 & -\lambda_{2} \\
-\mu_{2} & 0 & c_{3} & 0 & 0 & -\lambda_{3} & -\lambda_{1} \\
-\mu_{3} & 0 & 0 & c_{4} & -\lambda_{1} & -\lambda_{2} & 0 \\
0 & -\mu_{3} & 0 & -\mu_{1} & c_{5} & 0 & 0 \\
0 & 0 & -\mu_{3} & -\mu_{2} & 0 & c_{6} & 0 \\
0 & -\mu_{2} & -\mu_{1} & 0 & 0 & 0 & c_{7}
\end{array}\right)^{-1}-
$$

$$
-\left(\begin{array}{lllllll}
1 & 0 & 0 & 0 & 0 & 0 & 0 \\
0 & 1 & 0 & 0 & 0 & 0 & 0 \\
0 & 0 & 1 & 0 & 0 & 0 & 0 \\
0 & 0 & 0 & 1 & 0 & 0 & 0 \\
0 & 0 & 0 & 0 & 1 & 0 & 0 \\
0 & 0 & 0 & 0 & 0 & 1 & 0 \\
0 & 0 & 0 & 0 & 0 & 0 & 1
\end{array}\right) \cdot\left(\begin{array}{c}
\tau_{1} \\
\tau_{2} \\
\tau_{3} \\
\tau_{4} \\
\tau_{5} \\
\tau_{6} \\
\tau_{7}
\end{array}\right)\left(\begin{array}{c}
\tau_{1}^{2} \\
\tau_{2} \\
\tau_{3} \\
\tau_{4} \\
\tau_{5} \\
2 \\
\tau_{6} \\
\tau_{7}
\end{array}\right)
$$

Using (6) and (11) we will find probability of finding of the source-protection-Wednesday system in each of possible states by means of the system of the linear differential equations which is written down in a matrix look:

$\left[\begin{array}{c}P_{1}^{\prime}(t) \\ P_{2}^{\prime}(t) \\ P_{3}^{\prime}(t) \\ P_{4}^{\prime}(t) \\ P_{5}^{\prime}(t) \\ P_{6}^{\prime}(t) \\ P_{7}^{\prime}(t)\end{array}\right]^{T}\left[\begin{array}{c}P_{1}(t) \\ P_{2}(t) \\ P_{3}(t) \\ P_{4}(t) \\ P_{5}(t) \\ P_{6}(t) \\ P_{7}(t)\end{array}\right]\left[\begin{array}{ccccccc}-c_{1} & \lambda_{1} & \lambda_{2} & \lambda_{3} & 0 & 0 & 0 \\ \mu_{1} & -c_{2} & 0 & 0 & \lambda_{3} & 0 & \lambda_{2} \\ \mu_{2} & 0 & -c_{3} & 0 & 0 & \lambda_{3} & \lambda_{1} \\ \mu_{3} & 0 & 0 & -c_{4} & \lambda_{1} & \lambda_{2} & 0 \\ 0 & \mu_{3} & 0 & \mu_{1} & -c_{5} & 0 & 0 \\ 0 & 0 & \mu_{3} & \mu_{2} & 0 & -c_{6} & 0 \\ 0 & \mu_{2} & \mu_{1} & 0 & 0 & 0 & -c_{7}\end{array}\right]$.

The system of the equations (14) decides under the following entry conditions: $P_{1}(0)=1, P_{2}(0)=1, P_{3}(0)=1$, $P_{4}(0)=1, P_{5}(0)=1, P_{6}(0)=1, P_{7}(0)=1$.

For a case when:

$$
\lambda_{1} \leq 100 \mu_{1}, \quad \lambda_{2} \leq 100 \mu_{2}, \quad \lambda_{3} \leq 100 \mu_{3}
$$

From system of the equations (12) we find:

$$
\tau_{1}=\frac{\mu_{1} \mu_{2} \mu_{3}}{\lambda_{1} \lambda_{2} \lambda_{3}\left(\mu_{1}+\mu_{2}+\mu_{3}\right)},
$$


In case to check operability of system of protection through time interval $\Theta_{l}$ (checks are absolutely reliable), then we will determine the parameter of process $\mu_{2}$ as follows:

$$
\mu_{2}=\frac{1}{\Theta_{1}-\frac{1}{\lambda_{2}}\left(1-e^{-\lambda_{2} \Theta_{1}}\right)},
$$

In case $\lambda_{2} \Theta_{1}<0,1$ then:

$$
\mu_{2}=\frac{2}{\lambda_{2} \Theta_{1}^{2}} .
$$

If availability of combustible material in electric network to control each $\Theta_{2}$ of year and to consider that checks are absolutely reliable, then:

$$
\mu_{3}=\frac{1}{\Theta_{2}-\frac{1}{\lambda_{3}}\left(1-e^{-\lambda_{3} \Theta_{2}}\right)} .
$$

In case $\lambda_{3} \Theta_{2}<0,1$ then:

$$
\mu_{3}=\frac{2}{\lambda_{3} \Theta_{2}^{2}} .
$$

We will substitute in a formula (16) of $\mu_{2}$ and $\mu_{3}$ value, received by means of formulas (18) and (20), then:

$$
\tau_{1}=\frac{\mu_{1} \cdot \frac{2}{\lambda_{2} \Theta_{1}^{2}} \cdot \frac{2}{\lambda_{3} \Theta_{2}^{2}}}{\lambda_{1} \lambda_{2} \lambda_{3}\left(\mu_{1}+\frac{2}{\lambda_{2} \Theta_{1}^{2}}+\frac{2}{\lambda_{3} \Theta_{2}^{2}}\right)} .
$$

In case $\mu_{1}<<\mu_{2}$ and $\mu_{1}<<\mu_{3}$, a formula (21) can be presented in the form:

$$
\tau_{1}=\frac{1}{0,25 \lambda_{1} \lambda_{2}^{2} \lambda_{3}^{2} \Theta_{1}^{2} \Theta_{2}^{2}} .
$$

When performing a condition:

$$
\tau_{1}=\sqrt{D_{1}} .
$$

The probability of ignition of combustible material in electric network during time of $t$ can be defined by a formula:

$$
F(t)=1-e^{-H_{1} t}
$$

where $H_{1}=\frac{1}{\tau_{1}}$.

If the condition (23) isn't satisfied, then $F(t)$ is defined as follows:

$$
F(t)=1-\sum_{i=1}^{7} P_{i}(t)
$$

where $P_{i}(t)$ are defined from the solution of system of the linear differential equations (14).

\section{Conclusion}

Ignition of combustible material on an electrical network can occur every time in case of coincidence in space and time of the next three accidental events: there was an accident on an electrical network which is energized; the system of protection which ensures fire safety of an electrical network refused actuating; there was a combustible material near the place of possible accident of an electrical network. Process of formation of ignition of combustible material on an electrical network because of its damage owing to appearance of dangerous electrical sources and a failure to actuating of system of protection can be described adequately by means of set of three uniform accidental Markov processes of $\alpha(t)$, $\beta(t)$ and $\xi(t)$ with the discrete number of statuses and the continuous time. The proposed system is based on equations (12) and (13), by which it is possible to define an average period of $\tau_{1}$ before the first ignition of combustible material on an electrical network and dispersion of this time of $D_{l}$. The system of the linear differential equations (14) which allows to estimate probability of ignition of combustible material on an electrical network during $t$ time from the frequency of appearance of damages to it, reliability of system of protection and periods of its diagnostics of $\Theta_{l}$, and also from the frequency of appearance of combustible material near possible sources of initiation of ignition of combustible material and intervals of time between its detection of $\Theta_{2}$, is received.

\section{References}

1. Federal Law of the Russian Federation from 22.07.2008 № 123-FL «Technical Regulation on the Requirements of Fire Safety» with Change comply with the Russian Federation Federal Law № 117-FL from 10.07.2012.

2. The Official Website of the Ministry of the Russian Federation for Civil Defense, Emergencies and Elimination of Consequences of Natural Disasters]. Available at: http://www.mchs.gov.ru/activitie s/stats/Pozhari/2016_god (accessed 30.05.2017).

3. A.P. Kovalev, O. Ya. Solenaya, Electricity, 11, 3645 (2014)

4. State Standard 12.1.004-91. Fire Safety. General Requirements. Moscow, Standartov Publ. (1992)

5. Federal Law of the Russian Federation from 21.12.1994. № 69-FL «About Fire Safety» with Change Comply with the Russian Federation Federal Law № 234-FZ from 13.07.2015.

6. A.P. Kovalev, O.Ya. Solenaya, Industrial power, 11, 43-46 (2013)

7. K. Verfondern, X. Yan, T. Nishihara, H.-J. Allelein, International Journal of Hydrogen Energy, 42(11), 7551-7559 (2017)

8. W. Chen, K. Zhou, S. Yang, C. Wu, Renewable and Sustainable Energy Reviews, 75, 98-105 (2017) 